\title{
Forest Fire Smoke Recognition Based on Gray Bit Plane Technology
}

\author{
Yinglai Huang ${ }^{1}$, Shaoqing Tian ${ }^{2}$, Xiaofang $\mathrm{Sun}^{3}$, Meng Gao ${ }^{4}$ and Linlin Dai ${ }^{5}$ \\ ${ }^{1,2,4}$ Information and Computer Engineering College, Northeast Forestry University \\ Harbin, China \\ ${ }^{3}$ Office of teaching affairs, Northeast Forestry University \\ Harbin, China \\ ${ }^{5}$ Dalian Vocational Technical College, Dalian, China \\ 'nefuhyl@nefu.edu.cn, ${ }^{2}$ tiansqing@163.com,3240845950@qq.com, \\ ${ }^{4}$ gaomeng0916@126.com, ${ }^{5} 762598870 @ q q . c o m$
}

\begin{abstract}
Traditional smoke recognition based on HSI model or frame differentiation method can not reach the effect of real-time processing due to large amount of computation and requirement of too many images. A kind of automatic method for recognizing forest fire smoke based on gray bit plane aiming at to improve the real-time performance is proposed, analyze the gray information of forest fire smoke and use method combining gray bit plane with adaptive area of forest fire smoke, first, build gray bit plane of smoke image, and then calculate combining with the characteristics reflected by smoke information in each bit plane in order to improve the real-time performance and finally obtain the area of forest fire smoke. Experimental results show that the accuracy of recognizing area by analyzing and calculating gray bit plane is consistent with the traditional method, which takes a shorter time in processing and has actual application prospect to a certain degree.
\end{abstract}

Keywords: Smoke recognition, Bit plane, Mean filtering, Automatic recognition

\section{Introduction}

The occurrence of forest fire is mainly monitored by equipment combining far infrared and visible light in domestic at present [1], as the rapid development of image processing technology and increment of various kinds of algorithms for forest fire smoke recognition, it becomes possible to use image processing technology to rapidly and effectively monitor the occurrence of forest fire. The common smoke recognition methods include method based on neural network, method based on block segmentation and SVM, method based on least squares support vector machine, method based on wavelet transform and sparse optical flow and method based on multiple features, etc., in which method based on neural network uses three laser beams with different wavelength as the input of neural network and "fire smoke" as well as "factors nothing to do with fire" as the output of neural network, selects data from attenuation influence experiment on multi-wavelength laser caused by typical fire smoke and factors nothing to do with fire, and finally realize the smoke recognition [2-4]; method based on block segmentation and SVM uses block division and background difference method to 
obtain suspicious smoke areas according to the changing rules of color and brightness which smoke represents in RGB and HSV color space, uses main motion direction and ratio of high frequency and low frequency of energy in suspicious smoke and corresponding background area as the foundation to construct SVM classifier, and finally realize the smoke recognition, which is applicable to various fire detection scenarios [5-9]; method based on least squares support vector machine does a secondary segmentation to suspicious smoke area, uses color features, correlation coefficient and area changing as the input vector, which reduces the dimension of input vector, shortens the training dimension and finally achieve a faster classifying speed and a higher recognition rate [10-12]; method based on wavelet transform and sparse optical flow uses method combining block division and adaptive background to build brightness change model in RGB and HSV space as well as divides suspicious smoke area, then analyze the ratio of high frequency and low frequency of energy in suspicious background and smoke area by the two-dimensional discrete wavelet transform, finally realize the smoke recognition through tracing motivate area by sparse optical flow method [13-15]; method based on multiple features uses HSI space combining with actual smoke images to segment smoke, extract feature of suspicious pixel aiming at the smoke characteristics including correlate coefficient, irregular shape and translucency, method combining with each eigenvalue to recognize smoke improves the recognition rate [16-18].

Usually, neural network, frame difference and HSI model are selected to recognize forest fire smoke among the above smoke recognition methods. Method based on neural network needs large amount of data for training in the prophase which is applied to the single environment; network needs to be retrained when environment or scenario is changing, therefore, it can not satisfy the requirement of real-time to a certain degree; method based on frame difference can solve the inconvenience caused by scenario changing under the application of neural network, but it needs at least two images in order to get results, and the image can not shake, otherwise the frame difference method will fail; method based on HSI model can obtain the result by only one single image, but it needs to do some switch operation between RGB and HSI of the image in advance, so it takes a longer processing time. Method combining gray bit plane and adaptive area in this paper can detect the suspicious forest fire smoke area in a single image with less computation and realize the automatic recognition of it.

\section{Smoke Gray Plane Modeling}

An image of visible light contains large amount of information in which gray information can reflect the brightness degree of pixels. Image obtained by infrared device is grayscale image; therefore, gray information can be directly obtained from this kind of images. However, image obtained by visible light device is usually saved as format based on RGB

color model; it needs to calculate the R, G, B value of each pixel by formula (1) in order to get the gray value of each pixel in the RGB image. Formula (1) is defined as:

$$
G_{i}(x, y)=\frac{R+G+B}{3}
$$


In which, $G_{i}(x, y)$ is the gray value of the pixel point $i$ (with decimal format), R, G, B is respectively the value of red, green, blue.

The research can be continued only if the gray information of an image is obtained.

Grayscale bitmap divides the gray value according to binary values, for example, the corresponding binary value of a gray level with 255 is 1111111 , and a grayscale bitmap can be obtained by diving every bit of an image in binary format, which is shown in Figure.1. Bitmap can represent different kinds of information which can not be reflected by grayscale image, which is gray bit plane.

Detail steps to construct gray bit plane are described as follows:

(1) a piece of video is composed of frames with number $i$, each frame has gray levels with number $2^{k}$. The gray value of pixel point $(x, y)$ is $G_{i}(x, y)$ which can be defined as:

$$
G_{i}(x, y)=b_{k} 2^{k}+b_{k-1} 2^{k-1}+\cdots+b_{1} 2+b_{0}
$$

In which, $b_{k}=0$ or $b_{k}=1,0 \leq k \leq K-1$.

(2) in binary format, each pixel is composed of K bits, image composed of value $b_{k}$ of the same bit per pixel is the corresponding gray bit plane image of bit with number $\mathrm{k}$, let $\mathrm{K}=8$, then there exists 8 gray bit plane images corresponding to it, in which the gray bit plane in lowest level is $g_{0}(x, y)$ which mainly reflect the detail information of an image and the highest one is $g_{7}(x, y)$ which mainly reflect the overall information of an image. The 8 gray bit plane images of forest fire smoke based on visible light are shown in Figure 1. Among which Figure 1.A is the original image and corresponding gray image; Figure 1.B is the bitmaps of low level from first to fourth; Figure1.C is the bitmaps of high level from fifth to eighth.

It can be seen that the detail information of an image reflected by gray bit plane in different levels is various.
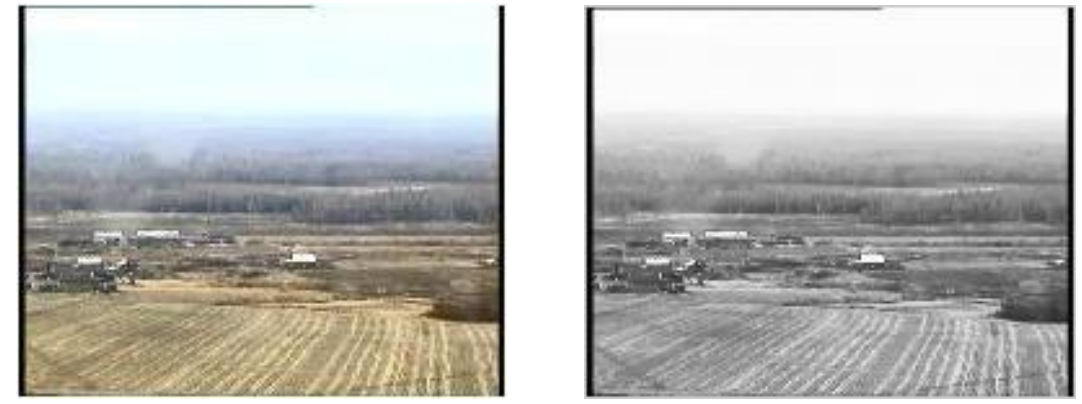

A. The original image and gray image 

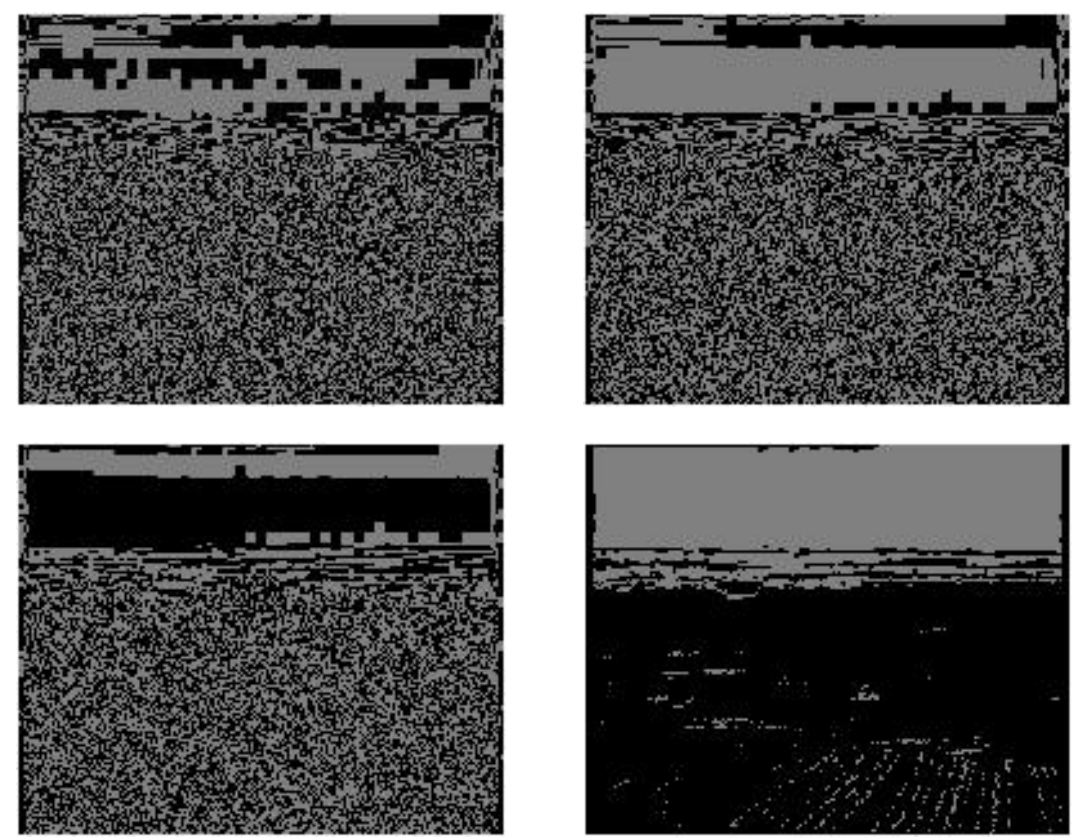

B. The first to the fourth bitmap
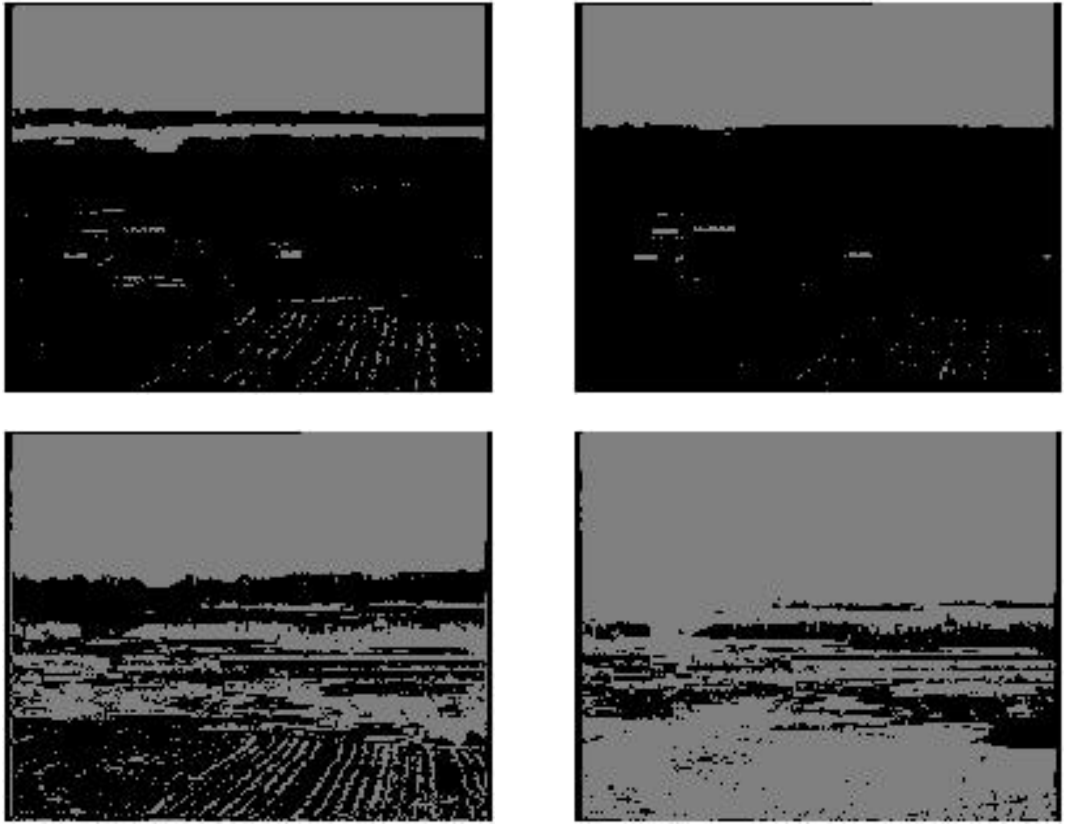

B. The fifth to the eighth bitmap

Figure 1. Gray bit plane graph

\section{Suspicious Forest Fire Smoke Region Detection Method based on Bit Plane Graph}

Information of forest fire smoke can be extracted according to the characteristics of gray bit plane we get, and in order to use information of high level in an image more accurate 
before extraction, it needs to weaken information of low level and use measures of stabilize or smooth information of high level to preprocess the image [19-21].

This paper uses mean filtering to process the gray image [22]. The filter is a method commonly used in image denoising, which uses the average value of gray values of several pixels in the neighborhood to replace the gray value of the current pixel in order to reduce the sharp changes of gray value, first, set the image function as:

$$
Z(i, j)=T(i, j)+x(i, j)
$$

In which, $T(i, j)$ is the image information function, $x(i, j)$ is the noise function. Then, use the smooth formula to calculate which can be defined as:

$$
\tilde{Z}(i, j)=\frac{1}{N} \sum_{1}^{4} Z(i, j)=\frac{1}{N}\left(\sum_{1}^{4} T(i, j)+x(i, j)\right)
$$

In which, $N=4$, according to the analysis of probability and statistics, the noise variance is:

$$
D\left(\frac{1}{N} \sum_{1}^{4} x(i, j)\right)=\frac{1}{N^{2}} \sum_{1}^{4} D(x(i, j))=\frac{1}{N} \omega^{2}
$$

In which, $D$ is the variance operation, $\omega^{2}$ is the noise variance without processing of neighbor smoothing. The noise variance through processing of neighbor smoothing reduces $\mathrm{N}$ times in order to achieve the effect of noise reduction and image smoothing. Figure 2.A is the bit plane graph after being filtered, it can be seen that information of low level is effectively weakened.

As smoke is discrete and moveable, therefore, introduce the motion blurring operator [23] before filtering in order to simulate smoke better, Figure 2.B is the bit plane graph which has added the motion operator and the connectivity of smoke areas is improved.

The two image groups are respectively composed by the original gray image and corresponding bitmaps from first to eighth.
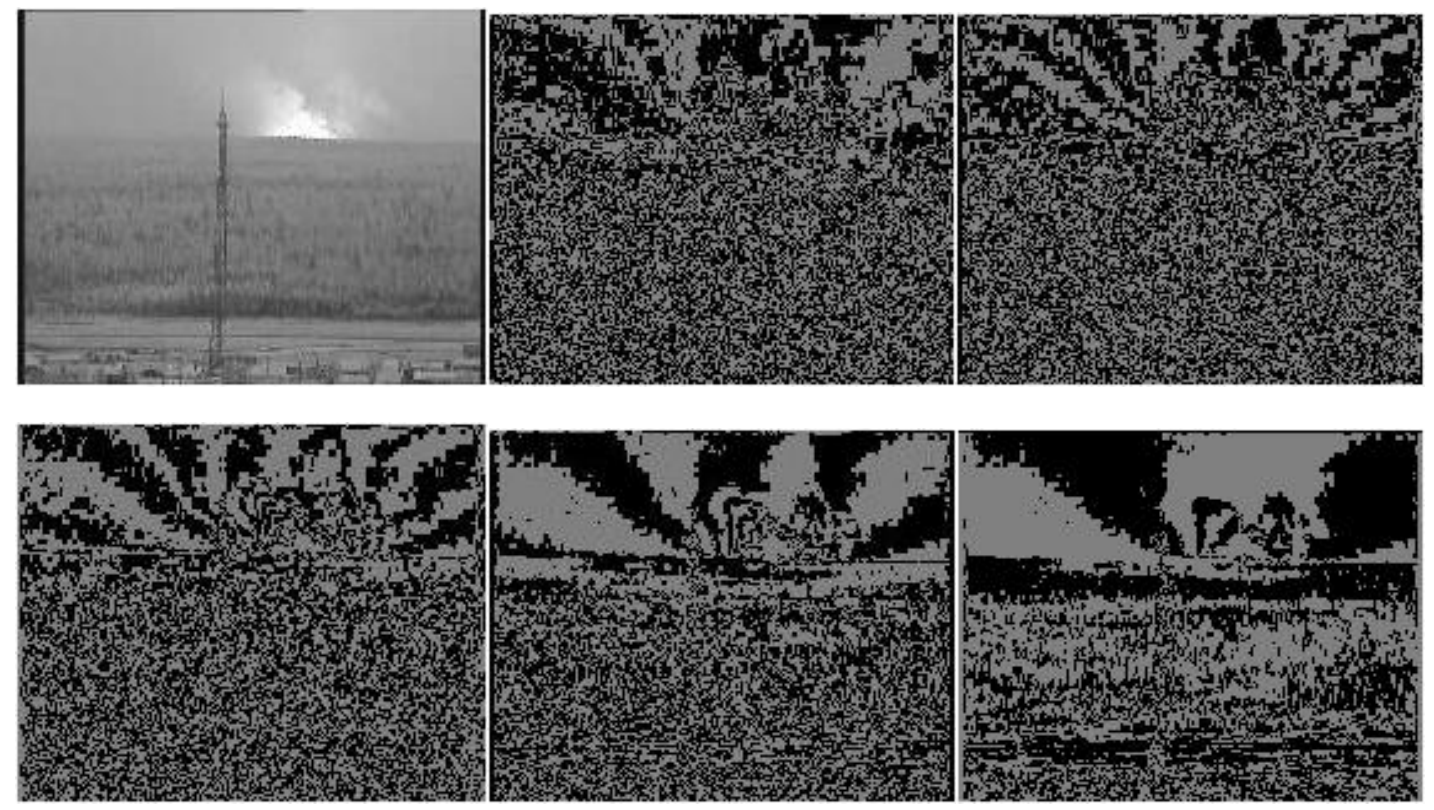


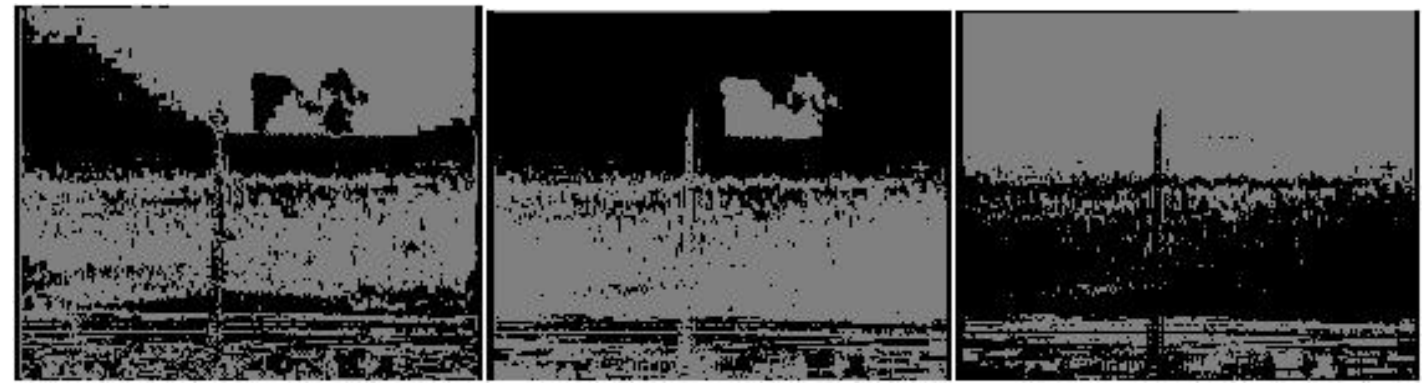

A. Process of mean filtering
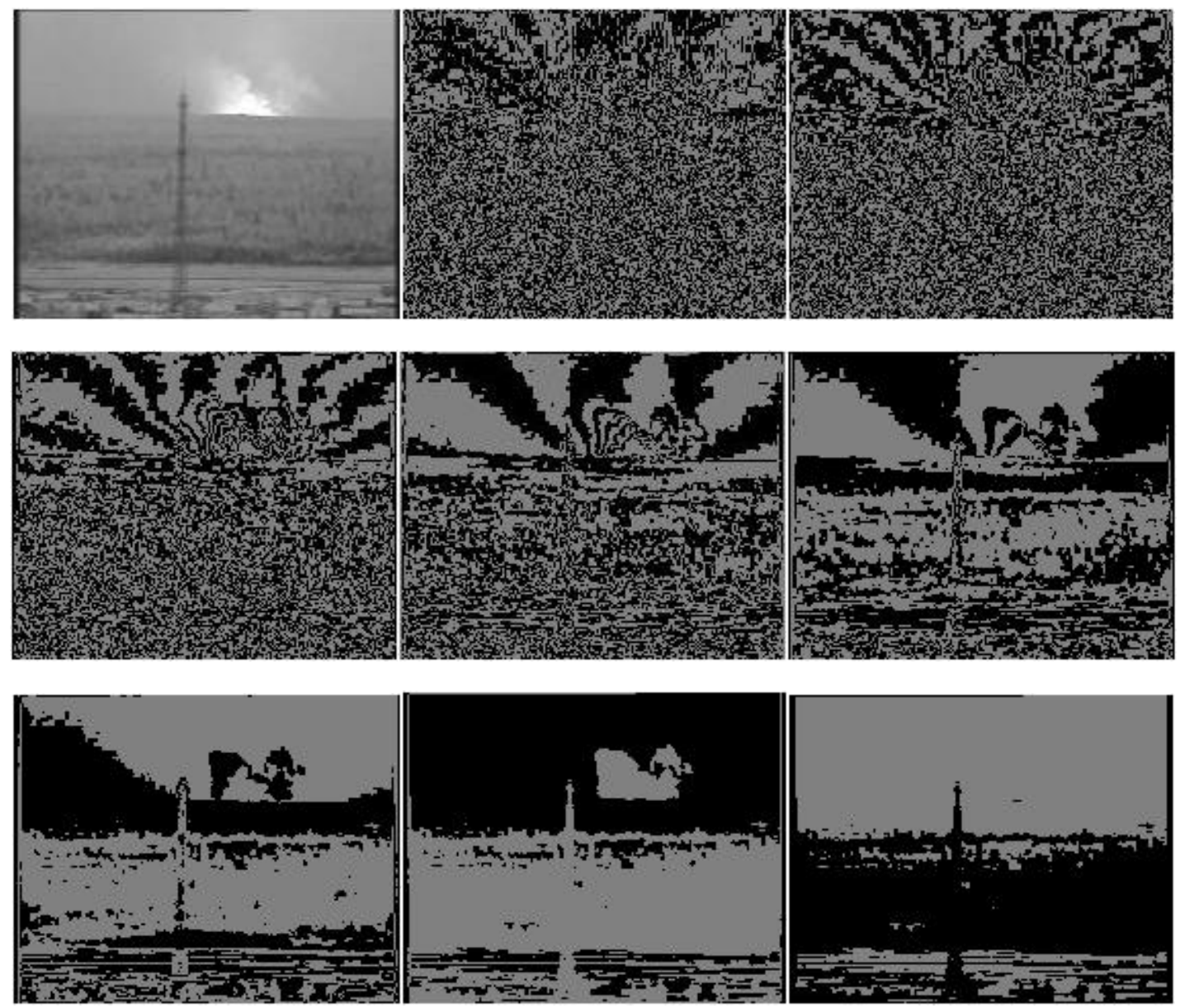

B. Process after adding motion blur operator

Figure 2. A plane graph under different conditions

The variance of detail model of an image will reduce $\mathrm{N}$ times in the process of denoising by mean filtering, which is suitable for bit plane with blur details. Combining with the analysis and processing in above, the smoke recognition model proposed in this paper can be defined as:

$$
\omega(x, y)=g_{K-2}(x, y) \bullet g_{K-1}(x, y)
$$




$$
\begin{gathered}
\psi(x, y)=\sum_{k=4}^{K-1}\left(g_{k}(x, y) \bullet \omega(x, y) \times 2^{K-k}\right) ; \omega(x, y) \neq 0 \\
\psi(x, y)=0 ; \omega(x, y)=0
\end{gathered}
$$

In which, $\omega(x, y)$ is the overall model of smoke, $\psi(x, y)$ is the detail model of smoke, $K=8$ in this paper. Forest fire smoke model can be quickly calculated by the method.

\section{Experimental Verification}

In order to validate the performance of this method, use notebook with Intel Core I3, 2GB DDR3 as hardware and Matlab7.0 as software to carry on the simulation experiment, use 10 forest fire videos obtained from a forest farm as the data source which can be divided into two categories: visible light image and far infrared image. Each video contains one kind of smoke scenario which includes forest fire in early and middle stage respectively in wind weather and windless weather. Figure 3 and Figure 4 are the whole process of far-infrared images and visible light images respectively, only processed bit planes whose levels are higher than 3 are listed as the gray bit plane information of low level is weaken by filtering and they do not contain the overall information of smoke.

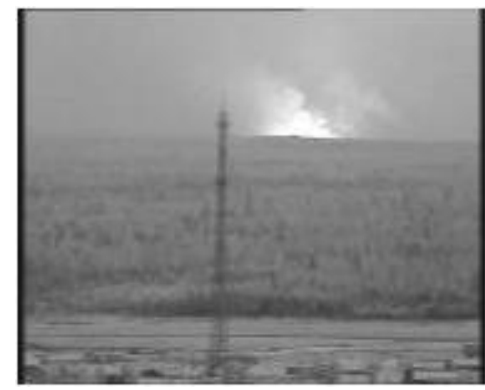

A. The original map

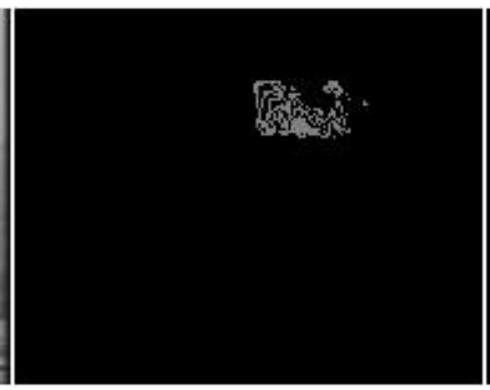

B. Third bitmap

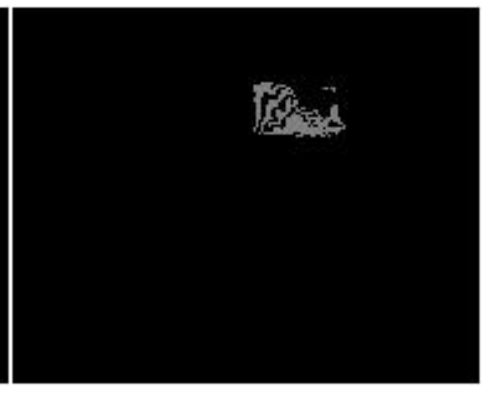

C. Fourth bitmap

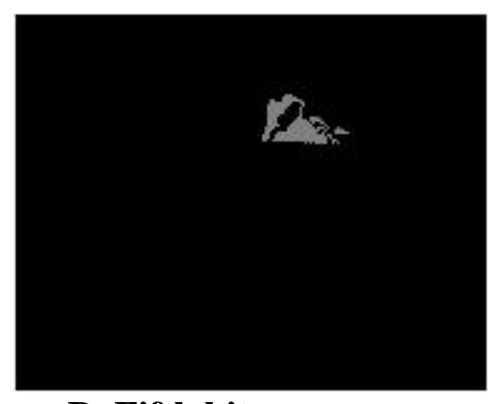

D. Fifth bitmap

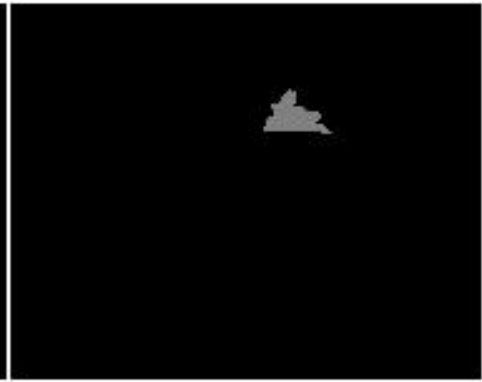

E. Sixth bitmap

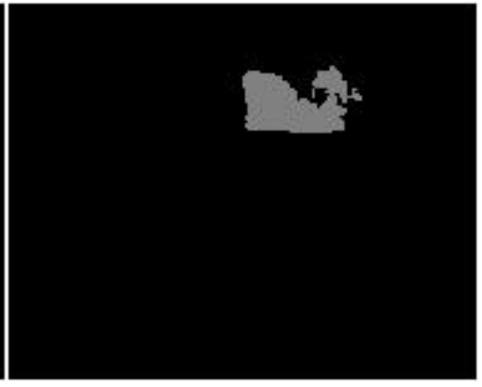

F. Seventh bitmap 


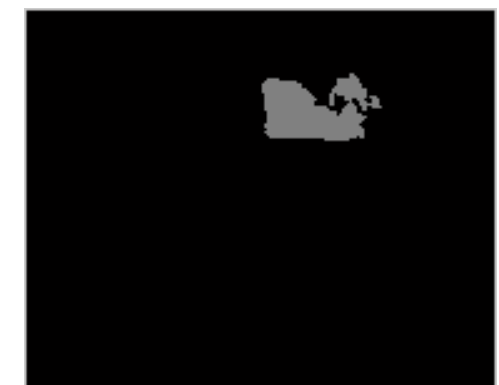

G. Eighth bitmap

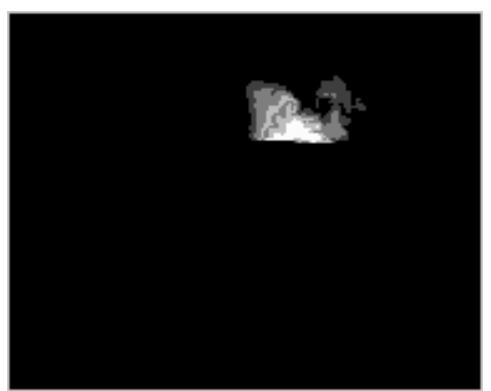

H. Smoke recognition graph

Figure 3. The results of far infrared video in this paper
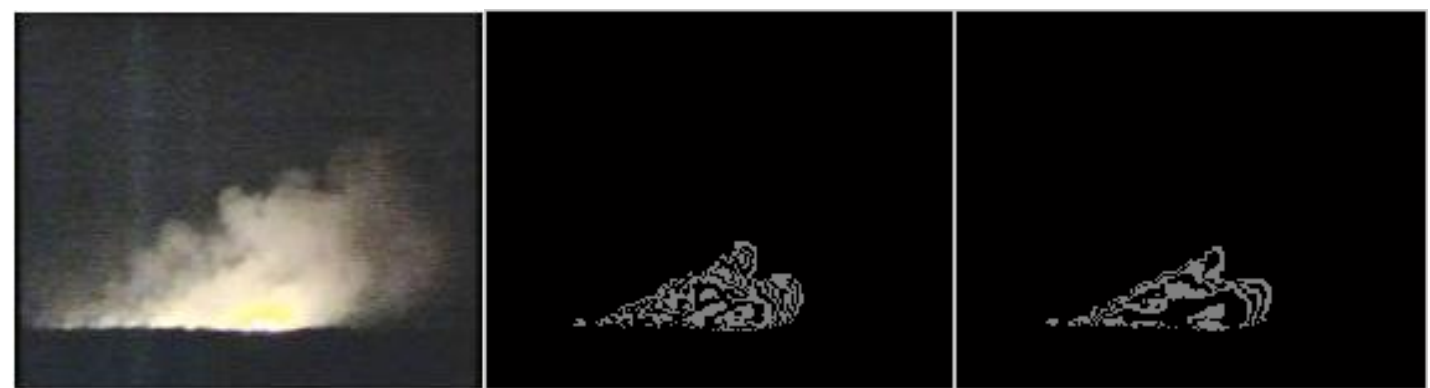

\section{A. The original map}

\section{B. Third bitmap}

\section{Fourth bitmap}
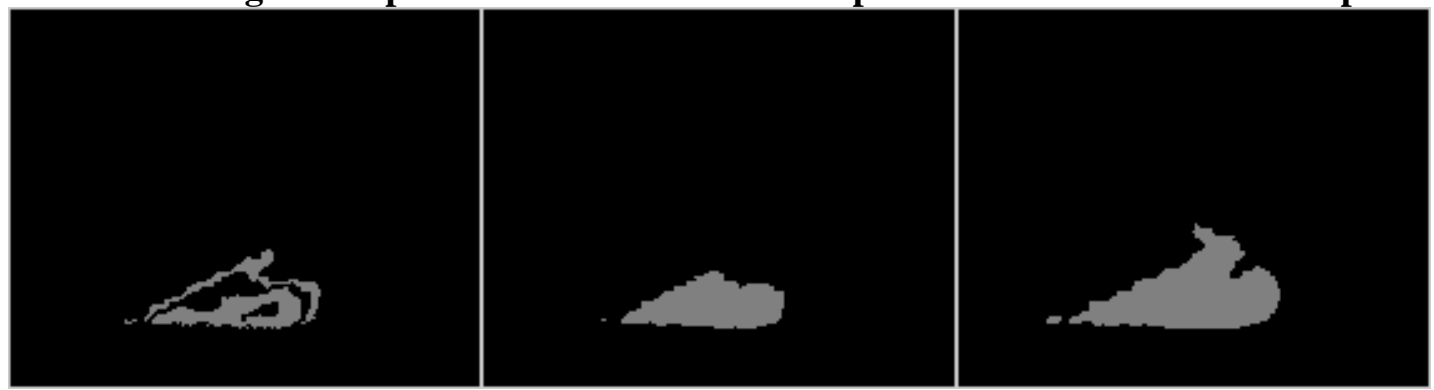

D. Fifth bitmap

E. Sixth bitmap

F. Seventh bitmap

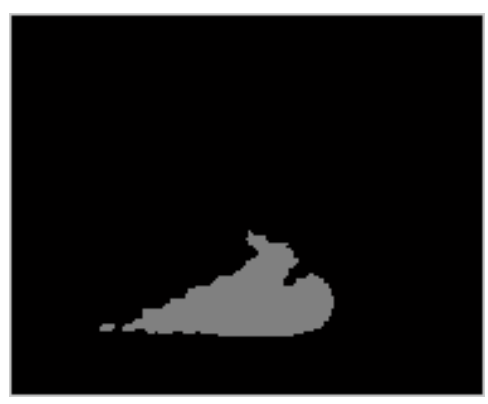

G. Eighth bitmap

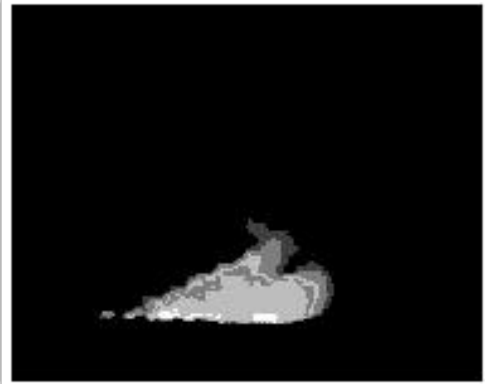

H. Smoke recognition graph

Figure 4. The results of visible light video in this paper

Figure 3.A is the original gray image, and Figure 3.B to Figure 3.G is respectively the bit plane graph corresponding with the third to eighth level, Figure $3 . \mathrm{H}$ is the smoke recognition image obtained by using the method proposed in this paper. 
In order to prove the method is better than the HSI method, carry out an experiment to compare with HSI model based on discriminate method, Table I is the processing time in different situations and Figure 5 is the comparison result,among which Figure 5.A is the infrared image got by infrared device; Figure 5.B is the gray image converted from infrared image; Figure 5.C is the smoke recognition image processed by HSI method; Figure 5.D is the smoke recognition images processed by method proposed in this paper. It can be seen form the result that the processing speed of method in this paper is faster than HSI method and the effect in smoke region recognition is generally consistent.

Table 1. Comparison result of two methods

\begin{tabular}{|c|c|c|c|c|}
\hline Image type & Fireproof period & $\begin{array}{c}\text { The number of } \\
\text { images (piece) }\end{array}$ & $\begin{array}{c}\text { The HIS method } \\
(\mathrm{s})\end{array}$ & $\begin{array}{c}\text { This method } \\
(\mathrm{s})\end{array}$ \\
\hline Windy weather & autumn & 120 & 3.483 & 2.310 \\
\hline In calm weather & autumn & 120 & 3.517 & 2.427 \\
\hline $\begin{array}{c}\text { At the initial } \\
\text { stage of fire }\end{array}$ & autumn & 120 & 3.521 & 2.353 \\
\hline $\begin{array}{c}\text { At the end of the } \\
\text { fire }\end{array}$ & spring & 180 & 4.256 & 2.973 \\
\hline
\end{tabular}

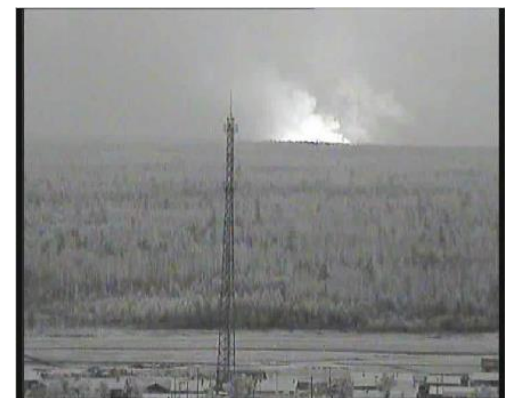

A. Infrared image

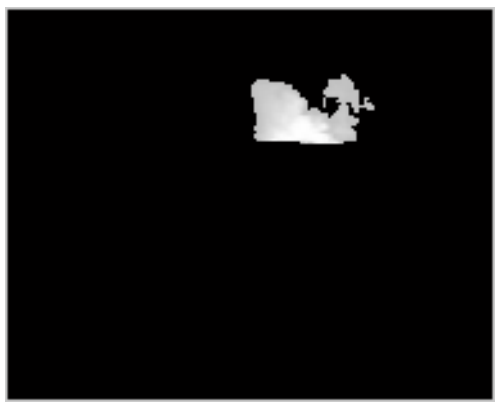

C.HSI method

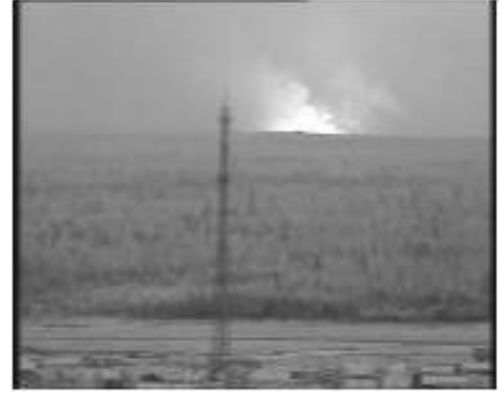

B. Grayscale image

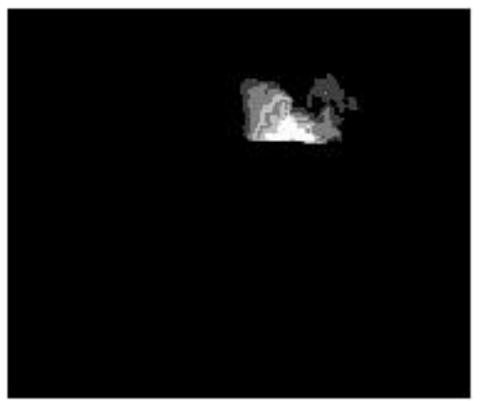

D. Method in this paper

Figure 5. Experiment result comparing with HIS

\section{Conclusion}

The goal of forest fire smoke recognition is to identify smoke timely and accurately. This paper uses gray bit plane to divide images firstly, and use mean filtering to smooth images 
and weaken information of low levels in order to guarantee the accuracy of information of high level, and then design the automatic recognition algorithm according to the smoke information reflected by different bit planes. Experimental results show that the method has lower amount of computation and it is able to satisfy the requirement of accuracy and realtime in automatic forest fire smoke recognition.

\section{Acknowledgements}

The work is supported by the Fundamental Research Funds for the Central Universities (DL11BB04),Supported by Natural Science Foundation of Heilongjiang Province of China(F201005), Technology Research Project of Heilongjiang Education Department (12523021), and funded by Graduate Education Innovation Project of Heilongjiang (JGXM_HLJ_2012008).

\section{References}

[1] Y. F. Zhao, B. Ling and L. C. Liu, "Ground fire automatic target detection based on infrared detection and tracking system design”, Journal of northeast forestry university, vol. 4, (2011).

[2] J. H. Zhao, J. Fang and X. M. Shu, "Fire smoke recognition method based on neural network", Journal of optics, vol. 9, (2003).

[3] L. W. Wang and Q. S. Liu, "Comprehensive optimization neural network algorithm", Micro computer and applications, vol. 5, (2012).

[4] M. Cottrell, M. Olteanu and F. Rossi, "Neural Networks for Complex Data", Künstliche Intelligenz, vol. 4, no. 26, (2012).

[5] Y. Hu, H. Q. Wang and T. W. Yao, "Video fire smoke recognition based on block segmentation and SVM", Computer simulation, vol. 9, (2012).

[6] D. Q. Yang, Z. K. Yang and S. Wang, "Smoke particle recognition system based on SVM algorithm", Journal of signal processing, vol. 2, (2002).

[7] S. A. Hosseini and H. Ghassemian, "A new fast algorithm for multiclass hyperspectral image classification with SVM”, International Journal of Remote Sensing, vol. 23, no. 32, (2011).

[8] W. H. Li, L. C. Xiao and Y. Wang, "A video smoke detection algorithm based on block", Journal of Jilin university (science edition), vol. 5, (2012).

[9] R. Huang and J. Wu, "Forest fire smoke image automatic identification of pattern classifier selection", Journal of Beijing forestry university, vol. 1, no. 34, (2012).

[10] J. Jia, H. Q. Wang and Y. Hu, "Based on least squares support vector machine (SVM) of fire smoke recognition algorithm", Computer engineering, vol. 2, no. 38, (2012).

[11] T. Falck, P. Dreesen and K. D. Brabanter, "Least-Squares Support Vector Machines for the identification of Wiener-Hammerstein systems", Control Engineering Practice, vol. 11, no. 20, (2012).

[12] X. Y. Wang and Z. K. Fu, "A wavelet- based image denoising using least squares support vector machine", Engineering Applications of Artificial Intelligence: The International Journal of Intelligent Real-Time Automation, vol. 6, no. 23, (2010).

[13] T. W. Yao, H. Q. Wang and Y. Hu, "Based on wavelet transform and sparse optical flow method of fire smoke detection", Computer engineering, vol. 6, no. 38, (2012).

[14] A. Pande and J. Zambreno, "The secure wavelet transform", Journal of Real-Time Image Processing, vol. 2, no. 7, (2012).

[15] A. Mian and R. Hartley, "Hyperspectral video restoration using optical flow and sparse coding", Optics Express, vol. 10, no. 20, (2012).

[16] J. Jia, "Based on a variety of characteristics of fire smoke detection algorithm", Value engineering, vol. 18, (2011).

[17] C. Yu and D. Hua, “An Early Fire Detection Method Based on Smoke Texture Analysis and Discrimination”, IEEE Congress on Image and Signal Processing, (2008).

[18] M. Yang, C. H. Zhao, Q. Pan and S. W. Zhang, "Multiple feature fusion based on wavelet analysis of smoke and spatial accuracy compensation of forest fire detection algorithm", Chinese journal of image and graphics, vol. 4, (2009).

[19] S. J. Ko, S. H. Lee and K. H. Lee, "Digital image stabilizing algorithms based on bit-plane matching", IEEE Trans Consumer Electronics, vol. 3, no. 44, (1998).

[20] S. J. Ko, S. W. Jeon and E. S. Kang, "Fast digital image stabilizer based on Gray-coded bit-plane matching", IEEE Transactions on Consumer Electronics, vol. 3, no. 45, (1999). 
[21] Y. M Yeh, H. C. Chiang and S. J. Wang, "Digital camcorder image stabilizer based on gray-coded bit-plane block matching", Opt. Eng, vol. 10, no. 40, (2001).

[22] S. Rakshita, A. Ghosh and B. U. Shankara, "Fast mean filtering technique (FMFT)", Pattern Recognition: The Journal of the Pattern Recognition Society, vol. 3, no. 40, (2007).

[23] T. Gao, P. Wang and C. S. Wang, "Feature Particles Tracking for Moving Objects", Journal of Multimedia, vol. 6, no. 7, (2012).

\section{Authors}

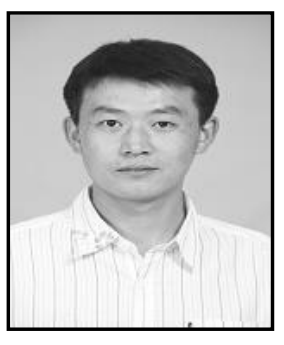

Yinglai Huang. He was born in October, 1978, who received his bachelor's degree in electronic information engineering (2003), master's degree in computer application technology (2006) and $\mathrm{PhD}$ in wood science and technology (2013) from Northeast Forestry University. Now he is mainly engaged in signal processing and computer intelligent processing direction as a full lecturer in Information and Computer Engineering College of Northeast Forestry University, he has published over 10 papers and hosted or participated in over 10 items of national and provincial projects.

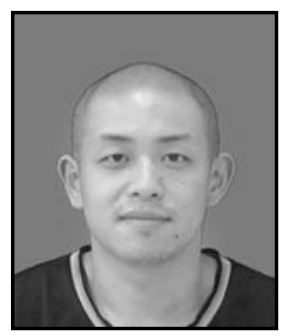

Shaoqing Tian. He was born in May 1983, who received his bachelor's degree (2007) from Inner Mongolia Agricultural University and master's degree (2011) from Northeast Forestry University. Now he is studying in Northeast Forestry University for the doctor's degree, and mainly engaged in image processing direction, he has published 5 papers.

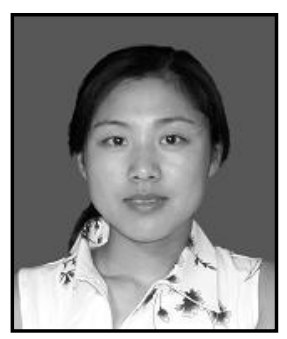

Xiaofang Sun. She was born in February, 1980, who received her bachelor's degree in computer science and technology (2003) and master's degree (2008) from Northeast Forestry University. Now she is studying in Northeast Forestry University for the doctor's degree and researching on forestry remote sensing and geographic information system as a lecturer.

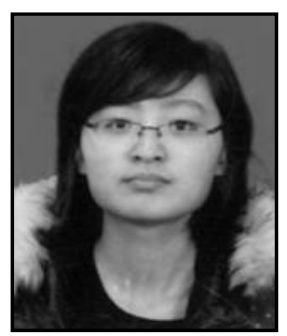

Meng Gao. She was born in September, 1989, who received her bachelor's degree in Information Management and Information System (2010) and master's degree in computer application technology (2012) from Northeast Forestry University. Now she is studying in Northeast Forestry University for the doctor's degree and researching on forestry information engineering, she has published 4 papers. 
International Journal of Signal Processing, Image Processing and Pattern Recognition Vol.6, No.6 (2013)

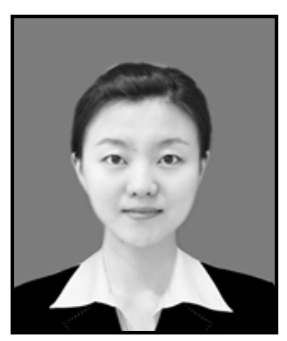

Linlin Dai. She was born in May 1988, who received her bachelor's degree (2011) and master's degree (2013) from Northeast Forestry University. 